\author{
Konstantinos Vadikolias \\ Ioannis Heliopoulos \\ Grigorios Tripsianis \\ Athanasios Achtaropoulos \\ Elias Homsioglou \\ Nickolaos Artemis \\ Haritomeni Piperidou \\ Ioannis Milonas
}

Received: 14 January 2002

Accepted in revised form: 22 April 2002

K. Vadikolias • A. Achtaropoulos

E. Homsioglou

Army General Hospital of Alexandroupolis,

Alexandroupolis, Greece

I. Heliopoulos (区) • H. Piperidou

Department of Neurology,

Democritus University of Thrace,

General Hospital of Alexandroupolis,

68100 Alexandroupolis, Greece,

e-mail: iiliop@med.duth.gr

Tel.: +30-5510-30344

Fax: +30-5510-31122

\section{G. Tripsianis}

Department of Medical Statistics,

Democritus University of Thrace,

Alexandroupolis, Greece

N. Artemis • I. Milonas

Department of Neurology,

Aristotle University of Thessaloniki,

Greece

\title{
Headache-related work disability in young men
}

\begin{abstract}
Based on the knowledge that migraine and tension-type headache (TTH) are associated with reduced effectiveness at work and impairment of function in social roles, we studied the different influences that these primary headaches have on work in a specialized and homogeneous population. We studied 140 consecutive male patients, aged $18-35$ years, attending an outpatient headache clinic at the Neurology Department of an Army General Hospital. Using International Headache Society (IHS) criteria, 60 patients were diagnosed with migraine and 80 patients with TTH. The impact of headache on work during the preceding 2 months was assessed using a selfadministered questionnaire, based on MIDAS. Two parameters of work disability were derived: the lost work days (LWD) and the days with reduced effectiveness while being at work (REWD). Of 142 LWD due to headaches, 95 (66.9\%) were attributable to migraine and $47(33.1 \%)$ to TTH $(p<0.001)$. Of 490 REWD, 120
\end{abstract}

(24.4\%) were reported by migraineurs and $370(75.5 \%)$ by TTH patients $(p<0.001)$. The number of LWD in migraine was significantly higher $(p<0.001)$ than in TTH group; the number of REWD in TTH group was significantly higher $(p<0.001)$ than in migraine group. Pain intensity was the main factor contributing to disability at work in migraine group $(p<0.001)$, in contrast with TTH group in which there was no statistical difference $(p>0.05)$ between pain intensity and duration of pain. Patients with migraine were much more likely to report actual lost workdays because of headache whereas TTH was responsible for the largest proportion of decreased work effectiveness. Assessing headache severity with an objective method (i.e. questionnaire) may improve headache care and lead to proper treatment decisions. Special attention must be given to particular populations.

Key words Migraine $\cdot$ Tension-type headache $\cdot$ Worn effectiveness

\section{Introduction}

People experience headache more than any other type of pain [1]. However, headache is a heterogeneous condition that results in a spectrum of disabilities within and among different individuals [2]. Headaches vary in their individual impact from mild pain with no disability, to severe pain with prolonged incapacitation [3]. During an attack, headacherelated disability can be severe and imposes a considerable aggregate burden on the sufferer and on his work environment [4]. 
Headache accounts for over 10 million physician visits annually in the US, and health statistics estimate a total amount over US \$13 billion a year due to headache-related missed work days in US, 1.1 billion in the UK, 1.2 billion in the Netherlands and 1.1 billion in Spain [5, 6]. At the same time, migraine and tension-type headache (TTH) remain underestimated and under-diagnosed, so there is an underassessment of headache disability [5, 7]. Headache-related disability affects most aspects of daily life, including employment, household work, and non-work activities. Previous studies have shown the importance of assessing disability in clinical practice $[8,9]$. Since headache sufferers often stay at work during attacks with reduced effectiveness, a simple measure of missed work days does not capture all work-related disability. As a result, both individual and societal impacts are underestimated [10]. The prevalence of migraine and TTH varies with age, gender, race, socioeconomic status and geographic location and this evokes problems in comparison of the results from different studies with heterogeneous populations.

The objective of this study was to measure the effect of the most common types of primary headache, migraine and $\mathrm{TTH}$, in a specialized, young male, race-homogeneous population, so as to achieve better differentiation between migraine and TTH disability on work effectiveness. According to our knowledge, there are no previous published studies focused on young men.

\section{Subjects and methods}

\section{Participants}

We considered a sample of 220 consecutive male patients, aged 18-35 years, who were referred to headache outpatient clinic of a military hospital. All patients had neurological and physical examinations. Further investigation (e.g. hematological and biochemical examinations or neuro-imaging procedures) was performed when needed. Patients with other health problems during the previous two months were excluded from the study. All patients were being treated with non-steroidal anti-inflammatory drugs, and only rarely with triptans, prescribed by general doctors during the period before their reference to our clinic. Using International Headache Society (IHS) criteria [11, 12], 60 patients were included in the "migraine group" (group A) and 80 patients in the "tension-type headache group" (group B). Patients with mixed types (migraine and tension-type attacks) were classified according to the general rule that the attacks should be coded as the type listed first in the classification [10]. Patients with other types of headache ( 80 patients) were excluded from the study.

\section{Setting and design}

The impact of headache on work during the last 2 months was assessed. The patients self-administered a questionnaire derived from the work disability section of MIDAS (migraine disability assessment) scale. The MIDAS questionnaire is a reliable, accurate and easy to use tool for investigating headache-related disability $[9,13]$. In the present study, two questions from MIDAS were asked:

- On how many days in the previous two months did you miss work because of your headaches?

- On how many days in the previous two months was your productivity at work reduced (reduced effectiveness, decreased concentration, etc.) by half or more because of your headaches?

MIDAS questions that concerned household work, family and social non-work activities were excluded, because the objective of our study was to estimate the headache-related disability in the work environment. Additionally, these activities are usually compromised in daily life of some particular populations such as the patients of our study. Although previous work has shown that a 3month period was a short enough period for accurate recall, we used a 2-month period, which in combination to the official data registration, provided more accurate information.

Two parameters of work disability, the lost work days and the days with reduced effectiveness while being at work were derived:

- Lost work days (LWD) were derived from the questionnaire. We collected data about the number of days of hospitalization for headache for investigation or treatment purposes, and we determined the total number of the days in which the patients were free of tasks or duties because of headache, as all these were reported on the official hygiene book of each patient. We compared the answers on the self-reported questionnaire to the official data and if there were differences, we asked the patient to re-answer the questionnaire.

This second report was considered as the final.

- Reduced effectiveness work days (REWD) were estimated based on the answers to the questionnaire (reduced productivity during work time by half or more). Because of the special characteristics of the military workplace, we defined as "work time" all the activities during the week concerning the daily training program, work in technical support services and office work.

Finally, a third question concerned the major factors (maximum 2) contributing to disability at work for each type of headache, such as pain intensity, duration and number of concomitant symptoms (e.g. vomiting, nausea).

Statistical analysis was performed using SPSS v. 10.0. Continuous variables were expressed as mean and standard deviation (SD) or median and range, whenever appropriate, and categorical variables were expressed as percentages. Chi-square $\left(\chi^{2}\right)$ test was used to assess the association between categorical variables, and the Mann-Whitney test was used to determine differences of LWD and REWD between the two groups of patients. A $p$ value $<0.05$ was considered to be statistically significant.

\section{Results}

We studied the impact of headache on work in 60 men diagnosed with migraine and in 80 men with tension-type 
headache (TTH). This latter group comprised 41 men with episodic TTH (code 2.1 in the IHS classification), 25 men with chronic TTH (code 2.2) and 14 men with atypical TTH (code 2.3) not fulfilling IHS criteria for TTH.

Overall, the 60 migraineurs reported 95 lost work days (LWD) and 120 reduced-effectiveness work days (REWD) in the 2-month study period, while the $80 \mathrm{TTH}$ men experienced 47 LWD and 370 REWD (Fig. 1). Thus, for the 140 persons enrolled in the study, there were 142 LWD, of which $95(66.9 \%)$ were attributable to migraineurs and $47(33.1 \%)$ were reported by men with TTH $(p<0.001)$. For the 490 REWD overall, 120 (24.4\%) were due to migraine and 370 $(75.5 \%)$ were due to TTH $(p<0.001)$.

The median number of LWD was 1 (range, 0-6) in the migraine group over the two-month period and 0 (range, 0-7) in the TTH group. The Mann-Whitney test indicated that LWD in migraine were significantly higher $(p<0.001)$ than in TTH (Table 1). The median REWD was 2 (range, 0-6) in migraine group and 4 (range, 0-14) in TTH group. The Mann-Whitney test indicated that REWD in TTH were significantly higher $(p<0.001)$ than in migraine (Table 1$)$. The median total LWD and REWD, for all the participants of the study, was 4 bimonthly.

Among the special characteristics of headache related to lost work or reduced effectiveness in migraine group, pain intensity was reported by $85 \%$ of the patients, which was significantly higher $(p<0.001)$ than duration of pain (25\%) and concomitant symptomatology (18.3\%). In the TTH group there was no significant difference $(p>0.05)$ between pain intensity $(60 \%)$ and duration of pain (57.5\%) (Fig. 2).

Table 1 Number of work days lost (LWD) or of reduced effectiveness (REWD) in a 2-month period. Values are median (range)

\begin{tabular}{lccc}
\hline & Migraine (n=60) & TTH (n=80) & $p$ \\
\hline LWD & $1(0-6)$ & $0(0-7)$ & $<0.001$ \\
REWD & $2(0-6)$ & $4(0-14)$ & $<0.001$
\end{tabular}

$T T H$, tension-type headache

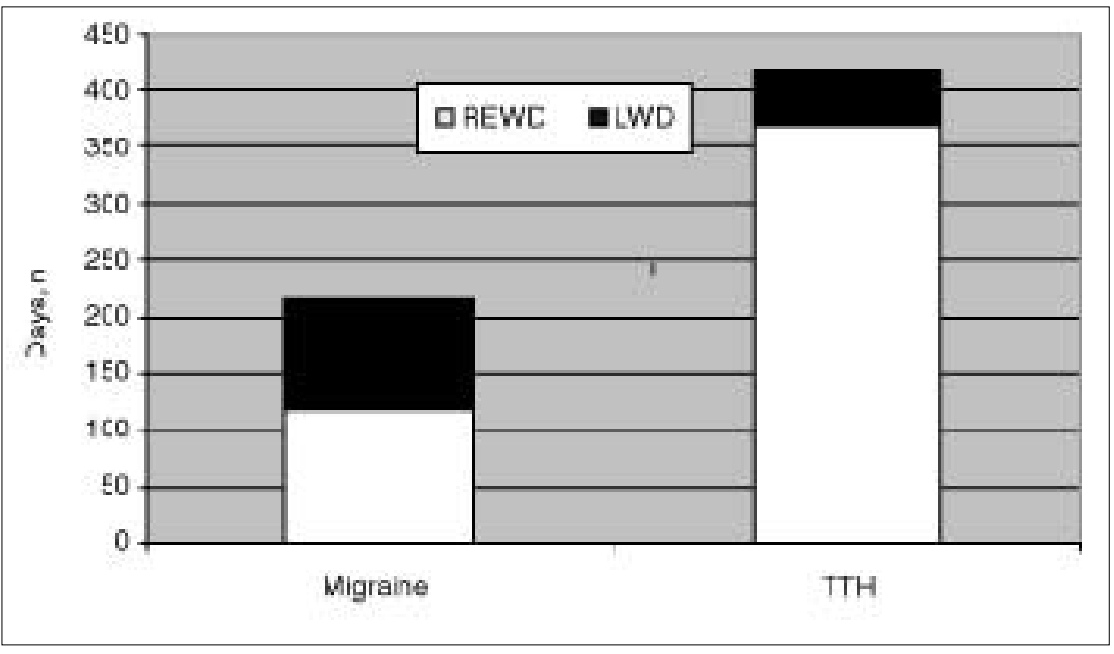

Fig. 1 Total number of work days lost (LWD) or reduced effectiveness (REWD) for 60 male migraineurs and 80 men with tension-type headache $(\mathrm{TTH})$, reported in a 2 -month period
Fig. 2 Characteristics of headache most responsible for absenteeism or reduced effectiveness at work, as reported by 60 migraineurs (black bars) and 80 patients with tensiontype headache (white bars)

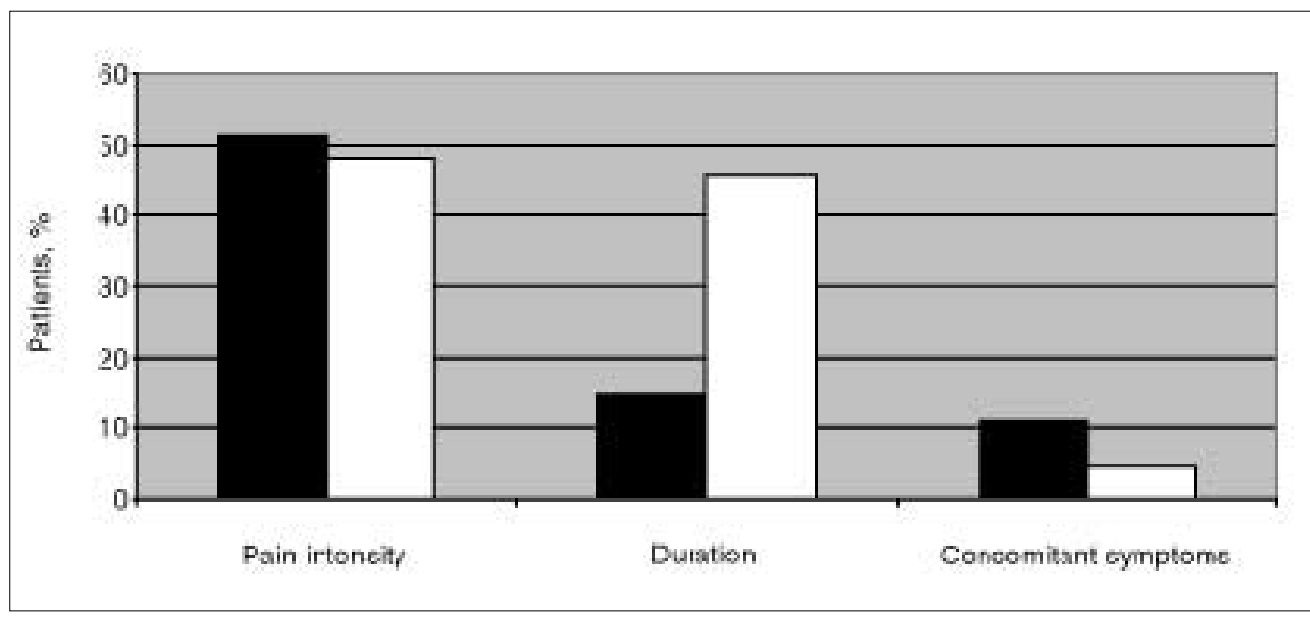




\section{Discussion}

The best knowledge about headache-related disability plays an important role in headache management according to the recent, evidence-based, consensus-driven US Headache Consortium Guidelines [1, 14]. Optimal management of migraine and TTH requires a systematic evaluation of the patient and the development of an individualized management plan [1, 14]. Work disability is an indicator of the effectiveness of different types of medications in headache management and, additionally, it is known that the indirect costs related to disability greatly exceed the medical care costs associated with the diagnosis and treatment of headache [6]. For these reasons, it is important to know the implication of each headache type in work disability. In our study we measured total LWD due to headache, we compared TTH and migraine on the basis of work-related disability and, finally, we investigated the main factors and headache characteristics responsible for disability. The special characteristics of our study population, i.e. gender, age and race "homogeneity", gave us the opportunity to make direct and objective comparisons of the study groups.

There are several reasons for the underestimation of headache disability, such as the fact that medical training tends to focus on diagnosis rather than on the consequences of disease. Recent studies consistently show that about three-quarters of migraine sufferers have a reduced ability to function during attacks with more than half reporting severe disability or the need for bed rest $[2,5,15,16]$. On the other hand, measuring severity is challenging, because of the episodic occurrence of attacks and variation in impact from one attack to the next. In the individual, headaches may vary in level of pain, duration, associated symptoms and disability [5, 13]. For all these reasons, the exact impact of headache on work is difficult to be measured with accuracy, and the comparison between studies is a difficult subject.

In our study, we recorded a higher number of total LWD in comparison to previous studies [9, 17-20]. Apart from the widely accepted view about the underestimation of disability, there are additional special reasons for this discrepancy. First, we used a short recall period (two months) that reduces the probability of wrong answers to the questionnaire (better memory of each attack). Evidence suggests that recall of pain experience may be more prone to error [19, 21] and as a natural consequence a large recall period increases the error probability. Second, the population of our study has special characteristics due to the conditions of the military environment (e.g. the exposure to many exogenous trigger factors for headache such as weather conditions, daylight, fast or missed meals, exertion and fatigue). The multifactorial trigger mechanisms also made the patients unlikely to eradicate attacks completely. Additionally, the personnel have more hours of work weekly. Third, our methodology, such as the direct interview and the classification from specialists, the confirmation of LWD reported on questionnaire by official data, and the inclusion of hospitalization days, gave us the opportunity to obtain more objective results.

Finally, the lack of a prophylactic treatment and the rare use of triptans by the patients in the study for the period preceding the visit to outpatient clinic was another reason for the higher estimated number of LWD in our study.

We found that the median number of LWD $x$ patient in tension-type headache was lower than in migraine. This finding is in accordance with recent surveys, which concluded that migraine subjects were more likely to report actual lost work days than those with TTH, although the total loss of work days per year due to tension-type headache is higher than that due to migraine $[17,18]$ because of its greater incidence in the general population. In the population of this study, subjects with tension-type headache reported more days of reduced effectiveness than those with migraine. This finding is in accordance with two studies conducted in a general population, where tension-type headache accounted for the large proportion of decreased work effectiveness $[10,17$, $18]$.

On the other hand, although it is a common belief that migraine significantly influences work ability, being an important social problem at the same time, tension-type headache seems to seriously affect patients' productivity and effectiveness at work.

The intensity of migraine pain is an established factor, and the main factor contributing to disability at work; it is also the most important factor for the male population [10]. This is in accordance with the results from our study. The duration and intensity of headache are equally associated with LWD in TTH [13].

In conclusion, patients with migraine headache were much more likely to report actual lost work days because of headache, whereas tension-type headache was responsible for a largest proportion of decreased work effectiveness. Special attention must be given to particular populations especially if they are exposed to multiple trigger factors. Further studies are needed to determine if early intervention for attack relief and a systematic prophylactic therapy would eliminate the headache-related work disability in such 


\section{References}

1. Silberstein SD, Rosenberg J (2000) Multispeciality consensus on diagnosis and treatment of headache. Neurology 54:1553

2. Stewart WF, Schechter A, Lipton RB (1994) Migraine heterogeneity. Disability, pain intensity and attack frequency and duration. Neurology 44[Suppl 4]:24-39

3. Lipton RB, Amatniek JC, Ferrari MD, Gross M (1994) Migraine. Identifying and removing barriers to care. Neurology 44[6 Suppl 4]:S63-S68

4. Lipton RB, Stewart WF, Goasby PJ (2001) Headache related disability in the management of migraine. Neurology 56[Suppl]:S1-S3

5. Holmes WF, McGregor EA, Dodick D (2001) Migraine related disability. Neurology 56[Suppl 1]:S13-S19

6. de Lissovoy G, Lazarus SS (1994) The economic cost of migraine: the present state of knowledge. Neurology 44[Suppl 4]:S56-S62

7. Lipton RB, Goasby PJ, Sawyer JPC et al (2000) Migraine: diagnosis and assessment of disability. Rev Contemp Pharmacother 11:63-73

8. Stewart WF, Lipton RB, Kolodner K et al (1999) Reliability of the migraine disability assessment score in a population-based sample of headache sufferers. Cephalalgia 19:107-114

9. Stewart WF, Lipton RB, Whyte J et al (1999) An international study to assess the reliability of Migraine Disability Assessment (MIDAS) Score. Neurology 53:988-994
10. Stewart WF, Lipton RB, Simon D (1996) Work related disability: results from the American Migraine Study. Cephalalgia 16:231-238

11. - (1988) Classification and diagnostic criteria for headache disorders, cranial neuralgias and facial pain. Headache Classification Committee of the IHS. Cephalalgia 8[Suppl 7]:19-28

12. - (2001) International Headache Society Members' Handbook, 2001-2002. Blackwell Science, p 65

13. Stewart Lipton RB, Dowson AJ, Sawyer J (2001) Development and testing of the Migraine Disability Assessment (MIDAS) Questionnaire to assess headache-related disability. Neurology 56[Suppl 1]:S20-S28

14. Lipton RB, Silberstein SD (2001) The role of headache-related disability in migraine management. Neurology 56[Suppl 1]:S20-S28

15. Stewart WF, Lipton RB, Celentano DD et al (1992) Prevalence of migraine headaches in the United States.

Relation to age, income, race and other socio-economic factors. JAMA 267:64-69

16. Sakai F, Igarash H (1997) Epidemiology of migraine in Japan. Cephalalgia 17:15-22

17. Rasmussen BK, Jensen R, Olesen J (1992) Impact of headache on sickness absence and utilisation of medical services: a Danish population study. J Epidemiol Community Health 46:443-446
18. Schwartz BS, Stewart WF, Lipton RB (1997) Lost work days and decreased work effectiveness associated with headache in the workplace. J Occup Environ Med 39:320-327

19. Van Korff M, Stewart WF, Simon DJ, Lipton RB (1998) Migraine and reduced work performance: a population-based diary study. Neurology 50:1741-1745

20. Michel P, Dartigues JF, Duru G, Moreay J, Salamon R, Henry P (1999) Incremental absenteeism due to headaches in migraine: results from the Mig-Access French national cohort. Cephalalgia 19:503-510

21. Stewart WF, Lipton RB, Simon D et al (1999) Validity of an illness severity measure for headache in a population sample of migraine sufferers. Pain 79:291-301

22. Lipton RB, Stewart WF, von Korf M (1997) Burden of migraine: societal costs and therapeutic opportunities. Neurology 48[Suppl 3]:S4-S9

23. Dasbach EJ, Carides GW, Gerth WC et al (2000) Work and productivity loss in the rizatriptan multiple attack study. Cephalalgia 20:830-834 